\title{
LA ECOAUDITORÍA EN UN CENTRO EDUCATIVO
}

\author{
Julián Mạ Cano Villanueva \\ IES Poeta García Gutiérrez. Chiclana (Cádiz)
}

\begin{abstract}
RESUMEN
El presente trabajo justifica el interés de una ecoauditoría para un centro de enseñanza de Secundaria y aporta una experiencia de trabajo en este proceso en un centro de enseñanza. La Ecoauditoría ha supuesto la búsqueda estrategias para la mejora medioambiental en el centro y la puesta en marcha de prácticas docentes encaminadas al desarrollo de hábitos cívicos y democráticos.
\end{abstract}

Palabras claves: currículo, Ecoauditoría, educación ambiental, mejora medioambiental.

\section{NRODUCCIÓN}

El presente trabajo pretende ofrecer una visión global de lo que significa una Ecoauditoría para un centro de enseñanza de Secundaria y aporta la experiencia derivada de haber trabajado en este proceso durante siete años, tras los cuales se puede decir que muchas cosas han cambiado en lo que se refiere al desarrollo de la educación ambiental en el centro y en las señas de identidad que actualmente definen al IES Poeta García Gutiérrez de Chiclana.

El proceso de Ecoauditoría ha supuesto para los agentes implicados una doble dimensión: por una parte la búsqueda de una mejora medioambiental y por otra la puesta en marcha de una práctica docente encaminada al desarrollo de hábitos cívicos y democráticos. La Ecoauditoría ha propiciado que se empiecen a hacer realidad algunos de los objetivos más idealistas del Proyecto de Centro.

\section{¿QUÉ SI GNI FI CA UNA ECOAUDI TORÍ A EN UN CENTRO EDUCATI VO?}

Se trata de un proceso que evalúa la calidad medioambiental de un centro escolar desde un punto de vista global, es decir, atendiendo a todos los aspectos relacionados con la misma: instalaciones, política de compras, gasto, medidas de eficiencia y ahorro, integración en el currículum, vinculación con el Proyecto de Centro y relación con las instituciones y organizaciones implicadas en la protección del medio ambiente.

La Ecoauditoría tiene como objetivos la mejora de la calidad ambiental del centro y la implicación en ella de los agentes que componen la Comunidad Educativa

En definitiva no es más que hacer realidad esa famosa frase de "Pensar a nivel global y actuar a nivel local". Con ello se pretende atacar el problema del deterioro del planeta desde lo cercano y singular para ir progresando en círculos concéntricos que 
nos hagan protagonistas de una de las luchas que debe afrontar nuestra especie en el siglo XXI: la búsqueda de un lugar más habitable para las generaciones venideras.

Podremos teorizar sobre lo mal que están las cosas en lo que a la degradación ambiental se refiere, culpabilizando a los gobiernos y organizaciones mundiales pero no conseguiremos avanzar nada en absoluto si no hay un compromiso individual desde el que adquirir una verdadera dimensión del problema y exigir, a los responsables de esas instituciones, un cambio

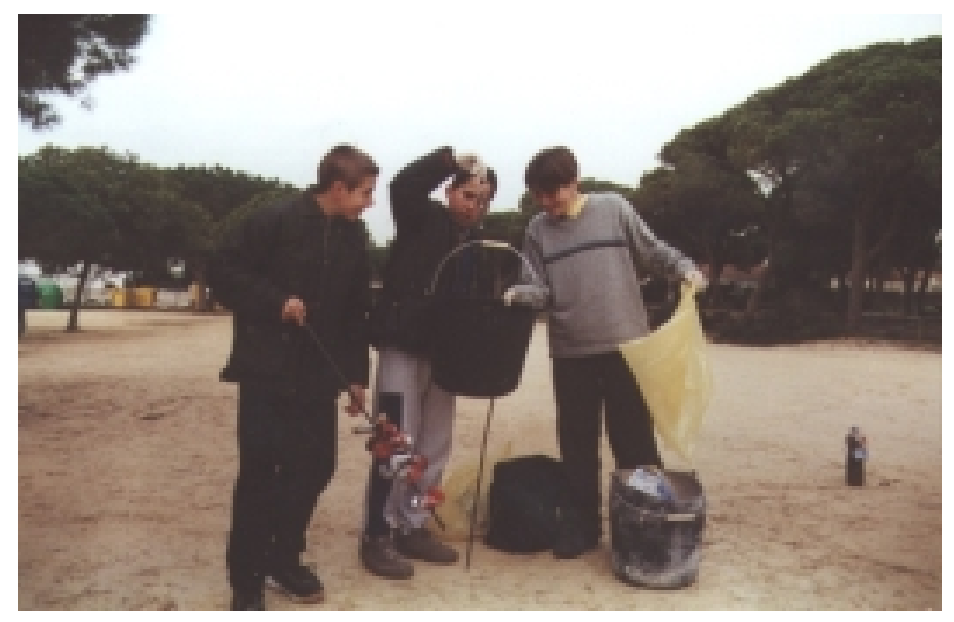
radical en el rumbo que, hoy por hoy, dirige la economía y la sociedad mundial (Mayer, 1998). Como señala el documento de Educadores por la sostenibilidad (2004), "se trata [...] de contribuir a formar ciudadanas y ciudadanos conscientes de la gravedad y del carácter global de los problemas y preparados para participar en la toma de decisiones adecuadas".

\section{¿PARA QUÉ SE HA DE REALI ZAR UNA ECOAUDITORÍ A?}

El proceso de Ecoauditoria se deriva de las Agendas 21 locales propuestas como forma de actuación en la Cumbre de Rio de Janeiro de 1992 (Naciones Unidas, 1992). Básicamente habría que generar un ámbito de discusión y decisión compuesto por los diferentes colectivos implicados que, aportando los intereses y sensibilidades diversos, analizarían la realidad ambiental del entorno próximo para determinar qué objetivos deben proponerse y qué camino debe seguirse para su consecución (Plan de Acción). Este esquema de funcionamiento es el que presenta el proyecto de Ecoescuelas:

a. Formación de un Comité Ambiental compuesto por repesentantes de cada estamento: profesorado, alumnado, padres-madres, PAS, Ayuntamiento y otras organizaciones locales.

b. Realización de una Ecoauditoría del Centro, que incluya su entorno más cercano.

c. Determinación de objetivos a corto, medio y largo plazo.

d. Diseño de un Plan de Acción encaminado a lograr esos objetivos.

e. Diseño de un Código de Conducta que, con frases concisas y breves, establezca unas normas generales de funcionamiento dentro del propio centro en aras de una mayor implicación en los problemas de nuestro entorno humano y natural.

f. Evaluación externa del Plan de Acción por un jurado de la asociación responsable de las Ecoescuelas en España, ADEAC (Asociación para la Defensa del Medio Ambiente y el Consumidor. 
En muchos centros se realizan actividades medioambientales pero de una forma esporádica y desconectada. Esto no está mal pero es insuficiente a todas luces. No debemos contentarnos con cubrir el expediente y cumplimentar en los documentos oficiales que hemos desarrollado esta materia transversal con la celebración del Día Mundial del Medio Ambiente o con una excursión a un espacio protegido. Es urgente tomar conciencia de que la escuela tiene una responsabilidad de primer orden en la salvaguarda del planeta (Edwards et al., 2004) y que sólo con programas específicos que impliquen a toda la Comunidad Educativa, podremos formar ciudadanos cada vez más comprometidos con la eliminación de las desigualdades.

No hay que pensar que el esquema de Ecoescuelas es el único posible ya que hay otras alternativas, pero sí hay que admitir que este proceso no puede ser algo exclusivo del profesorado o del Equipo Directivo, sino que tendrá que considerar la creación de un mínimo foro de discusión que agrupe todas las visiones y aglutine las posturas de los colectivos que se dan cita en el terreno de la educación.

Por tanto, es imprescindible una reflexión conjunta que nos lleve a descubrir el punto de partida de nuestro centro y determine las necesidades que tenemos, así como las acciones a realizar.

\section{¿CÓMO LLEVAR A CABO UNA ECOAUDITORÍ A?}

La metodología puede ser variable, no existiendo un único modelo válido. Hay que considerar que cada centro educativo tiene una realidad diferente. En cualquier caso, parece lógico que alguien dirija el proceso y perfile las líneas maestras que se deben seguir para realizar una Ecoauditoría. En el caso del programa Ecoescuelas, es el Comité Ambiental el eje vertebrador sobre el que gira esta responsabilidad. No quiere decir esto que sea el propio Comité quien tenga que llevar a cabo el estudio, sino que va a ser quien coordine, reúna, analice, saque conclusiones y difunda los resultados. La Ecoauditoría podemos dividirla en diferentes áreas, que podrían ser realizadas por grupos de alumnos/as) en el periodo de tiempo que se considerase. Los resultados de cada actividad, efectuada en las diferentes áreas de forma independiente adquirirán sentido para el centro, en el momento en el que el Comité Ambiental los analice y los compare, extrayendo las conclusiones. A partir de ahí, se podrían plantear estudios más concretos o diseñar otros.

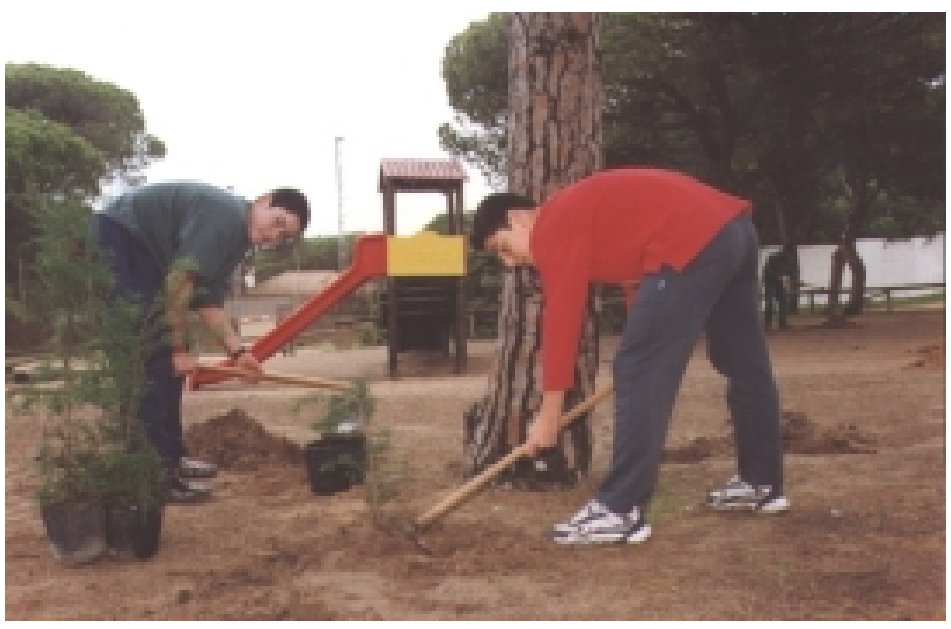

El funcionamiento de los centros de Primaria es diferente del de los de Secundaria. En el primer caso, el núcleo de actuación principal es la tutoría y en el segundo es el departamento. Esta diferencia, a priori poco llamativa, supone un matiz determinante 
que juega a favor de los centros de Primaria. La organización del trabajo se simplifica mucho si disponemos de un grupo de alumnado que pueda llevar actividades multidisciplinares de una forma continua y que no altere demasiado el funcionamiento habitual del centro. El tutor pasa la mayoría del tiempo con estos grupos y esto aporta una ventaja importante a la hora de implicar a este alumnado en la consecución de un objetivo común.

En el caso de Secundaria nos encontramos con un horario más cerrado, tanto del propio grupo como del centro. Existen muchos profesores que trabajan en el Equipo Educativo y la presión que se siente por el desarrollo del currículum es aún demasiado atosigante. No es fácil desarrollar actividades transversales que exijan una amplia disponibilidad horaria y un acuerdo común de quienes intervienen en todas las asignaturas que se imparten en el grupo. Además, los temarios y programaciones parten de los departamentos didácticos, que son quienes organizan cada materia y se ponen de acuerdo sobre lo que hay que realizar a lo largo del curso, incluyendo su implicación en las materias transversales que propone la LOGSE, algo que, dicho sea de paso, supone el último apéndice del último punto de su programación y suele ser un añadido en el que se cree poco y al que se le dedica menos esfuerzo todavía. Esta situación es aún más acusada en las enseñanzas post-obligatorias (Bachilleratos, Ciclos Formativos...) ya que, al menos en Secundaria, existe una hora de tutoría semanal con el grupo que puede venir muy bien para tratar alguna actividad medioambiental o algún aspecto de la Ecoauditoría. Esta hora permitirá trabajar a todos en la elaboración de la descripción de la calidad ambiental del centro. Se

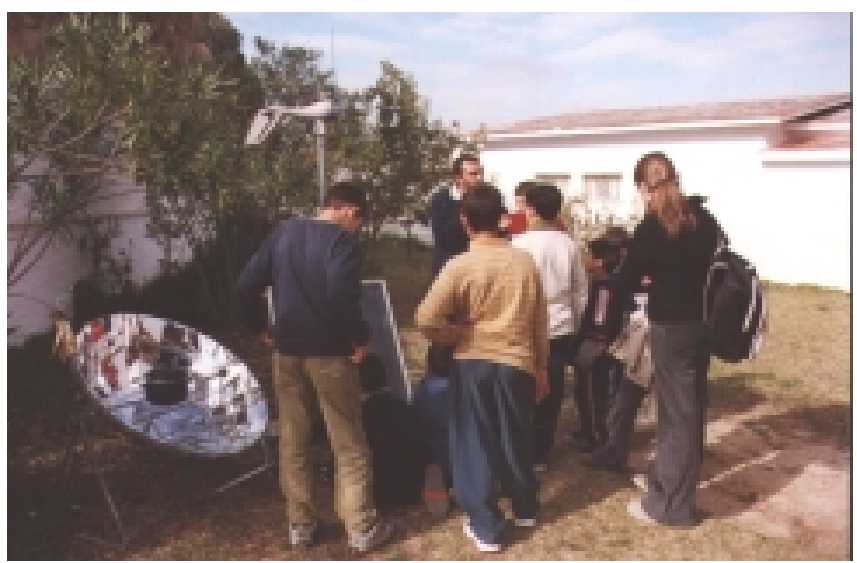
dispone también una hora semanal para que se reúnan el departamento de Orientación y los tutore. En este foro se puede planificar el trabajo, bajo las directrices del Comité Ambiental. Parece pues imprescindible la participación del departamento de Orientación en este proceso, incluyendo en su Plan de Acción Tutorial esta iniciativa.

El trabajo lo podemos estructurar de muchas formas pero no debemos olvidar determinados aspectos que deben ser abordados obligatoriamente:

a. Estado de las instalaciones: grifos, puntos de luz, enchufes, aparatos electrodomésticos, cuartos de baño, jardines, cocinas y comedores si los hubiere.

b. Medidas de ahorro y eficiencia energética: temporizadores, sistemas de ahorro de luz y agua.

c. Estudio del gasto del centro en aspectos como la electricidad, el agua, el papel, productos de limpieza y reprografía. 
d. Compras del centro: tipo de papel usado, grado de agresividad de los productos de reprografía y de limpieza y nivel de utilización de desechables.

e. Estudio de los sistemas de recogida selectiva del centro: evacuación del papel, del aluminio, de los plásticos, de las pilas, de los cartuchos de tinta de y de impresora.

f. Medida del nivel de implicación de los grupos en el ahorro energético y de agua, así como en la correcta evacuación de los residuos.

g. Compromiso del centro en la mejora de su entorno: municipio, comarca, estudiando las relaciones con las demás instituciones implicadas: Ayuntamiento, organizaciones sociales (ecologistas, conservacionistas), Delegaciones Provinciales de Medio Ambiente de Educación.

El periodo de tiempo en el que se puede desarrollar una Ecoauditoria también es variable. En ningún caso deberíamos sentirnos agobiados por cumplimentar documentos y debemos ser conscientes de que una tarea como ésta requiere aunar muchas voluntades. Nos lo podemos plantear como una labor a medio plazo, con un horizonte que no diluya en el tiempo su propio desarrollo, pero que deje madurar cada actividad por sí misma. Por ello, es mejor que se divida adecuadamente el trabajo aunque eso signifique una complicación mayor. El hecho de que intervenga la mayoría del alumnado, ya es en sí mismo un logro educativo. Además, nos va a facilitar el poder realizarlo en un tiempo menor. Podríamos pensar en abordar aspectos concretos en cada curso escolar, por ejemplo, un año estudiamos el consumo de agua, otro el consumo eléctrico y así sucesivamente.

A la hora de aportar los resultados, ni qué decir tiene, que debemos ser realistas. De nada nos va a servir presentar un panorama diferente del que hemos descubierto. Por más premios que nos puedan conceder, y con el programa de Ecoescuelas se puede conseguir una hermosa bandera verde, la realidad medioambiental del centro es algo que se percibe en cada momento $y$, de no reconocerla como es, podemos vernos sumidos en un estado de contradicción difícil de sobrellevar. En este sentido, somos conscientes de que todas las actuaciones a realizar en el centro deben incorporar criterios ambientales y plantear estrategias metodológicas acordes a las que propugna la educación ambiental (Conde et al., 2001).

\section{LA ECOAUDITORÍ A COMO UN PROCESO CONTI NUO}

En conexión con lo anterior, tampoco nos va a servir de mucho llevar a cabo una Ecoauditoría, lamentarnos de lo mal que estamos y no hacer nada más. Lo lógico es que, si hemos comenzado a trabajar y hemos descubierto nuestro punto de partida, nos propongamos un camino a seguir, con metas concretas que nos animen y nos hagan seguir adelante, con la idea de que estas mejoras nos van a reportar un medio más agradable y justo para todos.

Como todo proceso educativo se hace necesaria una evaluación, la nuestra encuentra en la metodología anterior las herramientas necesarias. No debemos dejar arrumbadas las actividades y cuestionarios que hemos rellenado entre todos, sino que 
deben ser remodeladas, corregidas y adaptadas a las nuevas circunstancias para utilizarse periódicamente a un ritmo más pausado y con una participación, si cabe, no tan global. De estas pequeñas Ecoauditorías continuas en el tiempo, se pueden encargar alumnos/as voluntarias (que siempre los hay, aunque parezca mentira) o grupos especiales, como pudiera ser el de Diversificación de 4으 de ESO. Con estos últimos, en concreto, hay que desarrollar una metodología especial y unos contenidos adaptados en las asignaturas de ámbito (científico-tecnológico y sociolingüístico), lo que unido a su reducido número proporcionan un medio eficaz y adecuado para llevar a cabo esta revisión de nuestro quehacer y mejora medioambiental.

El tema de la evaluación todavía está pendiente en muchos de los centros educativos, tal vez por la complejidad de su puesta en marcha o por el miedo a modificar determinados hábitos de conducta, sobre todo de los docentes, pero de ello depende, en buena medida, el buen funcionamiento de los mismos. Debemos partir de la idea de "evaluar para mejorar" y de que "todo es evaluable en educación". La Ecoauditoría es un mecanismo eficaz, colectivo y de retroalimentación positiva en lo que al desarrollo de la calidad ambiental de un centro se refiere. Su puesta en marcha nos hará tomar conciencia de lo que somos y sobre todo de lo que podemos conseguir entre los que formamos parte del entramado educativo

\section{¿Y DESPUÉS DE LA ECOAUDITORÍ A?}

Como hemos dicho anteriormente, no podemos contentarnos con haber realizado un estudio pormenorizado de nuestro centro, encuadernarlo y disponerlo para que engrose la prolija documentación que dormita en nuestros archivadores y discos duros. Por el contrario debemos considerar la Ecoauditoría como algo vivo a lo que recurrir, y sobre la que volver repetidamente en el futuro. La consecuencia directa debería ser una mejora sostenida, aunque fuera de poca intensidad. Debemos ser conscientes de que educar es un proceso que dura toda la vida y que en los tiempos que corren se ha convertido, a veces, en una especie de reto que exige valentía y mucho esfuerzo de los agentes implicados, por lo que no hay que arrojar la toalla si no observamos a nuestro alrededor una revolución verde que nos invada y nos aporte un mundo feliz en nuestra burbuja educativa. Las cosas no funcionan así, sino, más bien, a un ritmo lento y a veces cansino, con gran parte de los componentes de la Comunidad Educativa que viven de espaldas a lo que estamos llevando a cabo. Eso pasa con el medio ambiente, pero también con otras materias históricas que, después de décadas de estudio, mejora didáctica y adaptación curricular, no han conseguido enganchar a la mayoría del alumnado (ecuaciones, oraciones subordinadas o formulación química, pongo por caso).

El crecimiento de esta inquietud medioambiental estará asegurada si existe una voluntad de trabajar en este terreno por una parte de la Comunidad Educativa. Los efectos se irán viendo paulatinamente, de diferentes maneras: cantidad y calidad de actividades relacionadas con el medio ambiente, publicaciones específicas, iniciativas de esta índole en las que participa el centro, grado de consideración y colaboración con las instituciones, hábitos individuales de alumnos/as y profesores/as y hábitos familiares. Se debería notar también en otros aspectos como la limpieza general del 
centro o en la reducción de su gasto en energía, agua y papel, pero no siempre resulta sencillo contar con la colaboración de todos. Esto también es más fácil en un centro de Primaria, donde todas estas variables están más controladas desde las tutorías. En los centros de Secundaria -algunos con más de mil alumnos/as, una menor implicación del colectivo de padres y madres, y unas edades más difíciles de tratar-, en líneas generales, nos podremos sentir satisfechos si hemos conseguido llegar y comprometer, en cierta medida, a una parte de la Comunidad Educativa. Sea como fuere siempre que trabajemos con actividades variadas y, hasta cierto punto, lúdicas vamos a conseguir mejoras, que van a empezar por la propia dinámica de funcionamiento en lo que al hecho educativo se refiere. Como docente, tengo que decir que la forma de llevar a cabo el proceso de enseñanza-aprendizaje se ha visto profundamente transformado desde que un día,hace siete años, aquel incipiente Comité Ambiental decidió en el IES Poeta García Gutiérrez llevar a cabo una Ecoauditoría del centro. Desde entonces no hemos dejado de crecer, aunque haya muchas cosas que siguen mal, de lo que somos conscientes. En nuestra publicación anual, la revista "Nuestra Ecoescuela", que ya ha visto cuatro números consecutivos, tenemos una sección fija llamada "Fe de Ratas", en la que nos hacemos eco de todo aquello que no funciona bien y que no termina de avanzar convenientemente. Al margen de esto, hemos conseguido muchas cosas, quizá poco tangibles en ocasiones, pero que han traído como consecuencia que buena parte de los que formamos parte de esta Comunidad Educativa hayamos cambiado nuestro rumbo y nuestros hábitos medioambientales por un planeta más sano y habitable para todos.

Después de esta travesía por la educación ambiental, el resultado es que, para mucha gente, somos una referencia, algo que nos enorgullece pero nos hace más responsables, si cabe, en la idea de continuar en la brecha. Tanto en el municipio, como en el ámbito educativo más cercano, cada curso se espera algo de nosotros en el terreno de la formación medioambiental. Ya

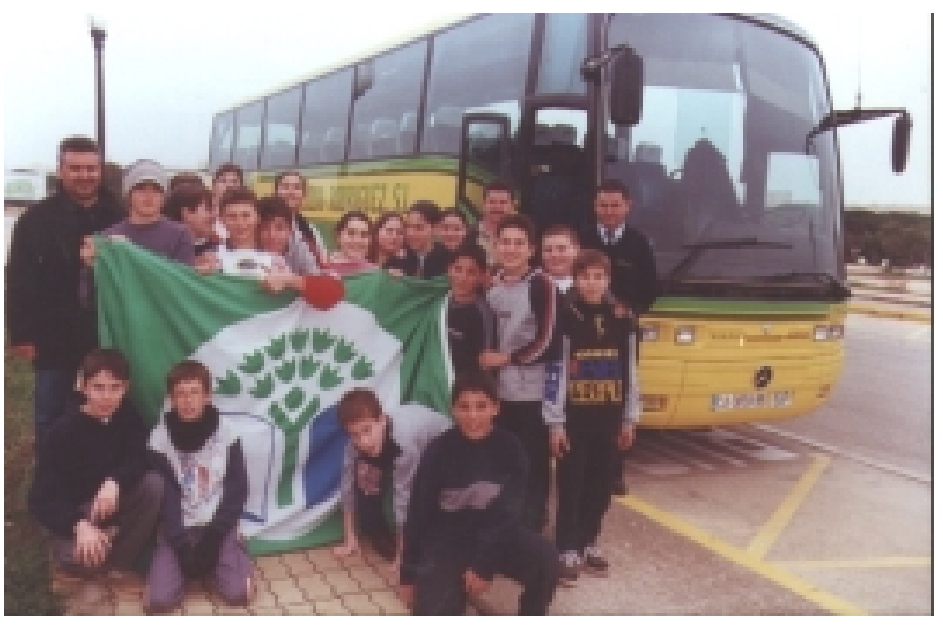
no podemos inhibirnos y retornar a la situación de partida porque hay muchos ojos que siguen nuestro trabajo y buscan en nosotros una respuesta afirmativa para comenzar su aventura. Sabiendo que sólo estamos al principio del camino y que lo conseguido significa un pequeño paso adelante queremos dejar constancia de que el esfuerzo realizado ha merecido la pena.

\section{REFERENCIAS}

CONDE, Mạ C., SÁNCHEZ, S. y DE PEDRO, J.Mạ (2001). Aportaciones de las experiencias de ecoauditorías en los Centros educativos a la didáctica de las 
ciencias. En. N. Elortegui et al., Actas de los XX Encuentros sobre Didáctica de las Ciencias Experimentales, pp 337-345. Universidad de La Laguna.

EDUCADORES POR LA SOSTENIBILIDAD (2004). Revista Eureka sobre Enseñanza y Divulgación de las Ciencias, 1(3). En línea en: http://www.apaceureka.org/revista/Volumen1/Numero_1_3Vol_1_Num_3.htm.

EDWARDS, M.; GIL, D.; VICHES, A. y PRAIA, J. (2004). La atención a la situación del mundo en la educación científica. Enseñanza de las Ciencias, 22(1), pp. 47-64.

MAYER, M. (1989). Educación ambiental: de la acción a la investigación. Enseñanza de las Ciencias, 16(2), pp. 217-232.

NACIONES UNIDAS (1992). Declaración de Rio sobre Medio Ambiente y Desarrollo. En línea en:

http://www.un.org/esa/sustdev/documents/agenda21/spanish/agenda21sptoc. htm. UNESCO. 